Supporting information for

\title{
Lasing from zero-dimensional perovskite and optical imaging
}

\section{applications}

Xun Sun, Zhenhua Gao, Yang Liu, Zifei Wang, Xue Wang, Weiguang Zhang, Baoyuan Xu, Xiangeng Meng*

${ }^{1}$ Key Laboratory of Processing and Testing Technology of Glass \& Functional Ceramics of Shandong Province, School of Materials Science \& Engineering, Qilu University of Technology (Shandong Academy of Sciences), 3501 Daxue Road, Changqing District, Jinan 250353, Shandong Province, China *Correspondence: mengxiangeng@gmail.com (X. M.)

This file includes:

Materials and Methods

Figures S1-S12 


\section{Materials and Methods}

Sample fabrication: $\mathrm{Cs}_{4} \mathrm{PbBr}_{6}$ microcrystals were fabricated with an antisolvent approach. In a typical procedure, $1 \mathrm{ml}$ dimethyl sulfoxide (DMSO) solution of $0.05 \mathrm{M}$ lead bromide $\left(\mathrm{PbBr}_{2}\right)$ and $0.2 \mathrm{M}$ cesium bromide $(\mathrm{CsBr})$ was injected into a glass vial. The glass vial is left open and put into a bigger vial. $5 \mathrm{ml}$ dichloromethane (DCM) was injected into the bigger vial to serve as the antisolvent. The glass vial was then sealed and put into a constant oven with temperature set at $40{ }^{\circ} \mathrm{C}$. The low boiling point of DMC makes it easy to create a high vapor pressure at low temperature. A miscible interface will be formed between DCM and DMSO, which will induce the growth of the crystals. The crystals were collected after $24 \mathrm{~h}$, and then washed with a mixed solvent of isopropanol (IPO) and DMSO (volume ratio: 3:1) several times. The resultant crystals were suspended in the IPO/DMSO solution and stored at room temperature for subsequent purposes. The microcrystals were spin-coated on silica glass substrates for micro-photoluminescence measurements.

The test mark embedded with "QLUT" characters in a silver film was fabricated on an ITO-coated glass substrate using electron beam lithography accompanied by electron beam deposition. The thickness of the silver film layer is $\sim 100 \mathrm{~nm}$.

Characterizations: X-ray diffraction patterns of the $\mathrm{Cs}_{4} \mathrm{PbBr}_{6}$ microcrystals were measured on a Bruker D8 advanced X-ray diffractometer equipped with graphitemonochromatized $\mathrm{Cu} \mathrm{K} \alpha$ radiation $(\lambda=1.5418 \AA)$. Surface morphologies and energy dispersive X-ray (EDX) analyses of $\mathrm{Cs}_{4} \mathrm{PbBr}_{6}$ microcrystals were examined with a 
scanning electron microscope (SEM, Carl Zeiss Gemini SEM500). Transmission electron microscope (TEM) images were recorded on a JEM 2100 transmission electron microscope with an accelerating voltage of $200 \mathrm{KV}$. Since the as-obtained particles are too large to allow for sufficient electron penetration, the specimen for TEM measurements is obtained by grinding the microcrystals to smaller particles and the HR-TEM is recorded by checking these smaller particles. The optical absorption spectrum was recorded on a UV-Vis-NIR spectrophotometer (UV-3600, SHIMADZU). PL spectra were measured with a fluorescence spectrophotometer (FLS1000, Edinburgh Instruments). The low-temperature PL spectra were obtained by using liquid-nitrogen cooling. The quantum yield of the $\mathrm{Cs}_{4} \mathrm{PbBr}_{6}$ microcrystals was evaluated with an absolute method using FLS1000 spectrometer equipped with an integrating sphere.

Optical measurements: In lasing experiments, the pump laser is from an optical parametric generator (EKSPLA, PG-401, $490 \mathrm{~nm}$ ) pumped by the third-harmonic output of a picosecond Nd: YLF laser (EKSPLA, PL-2250, $355 \mathrm{~nm}, 25 \mathrm{ps}$, and $1 \mathrm{~Hz}$ ). The pump laser was introduced into the home-built micro-photoluminescence system and focused onto the front surface of the crystal by an objective lens (20×, NA: 0.45). The PL signals were collected by the same objective lens and introduced through the micro-photoluminescence setup onto the entrance slit of a monochromator (Princeton Instruments, SP-2500) which is equipped with a CCD (Princeton Instruments, PIXIS 256). In speckle-free imaging experiments, an industrial camera (WP-UC1600, Work Power Inc.) was used to monitor the microcrystal and the SP-2500 monochromator 
equipped with the PIXIS 256 CCD was used to detect and analyze the PL signal. The PL signal emitted from the microcrystal was projected on the surface of the "QLUT" mark and a CCD (XM10-IR, Olympus) was used to capture the optical imaging of the "QLUT" target. 

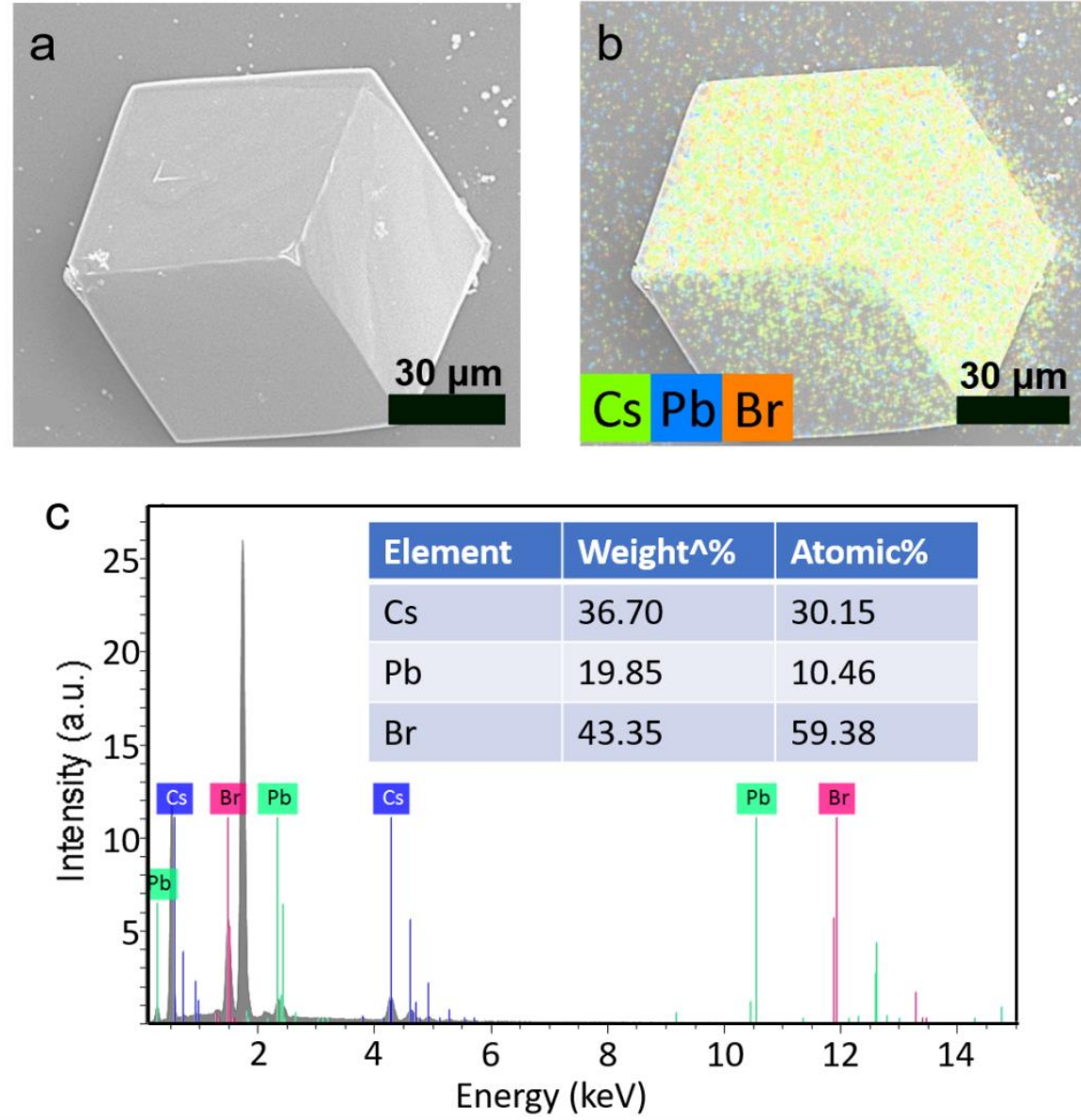

Figure S1. (a) SEM image of a selected $\mathrm{Cs}_{4} \mathrm{PbBr}_{6}$ microcrystal. (b, c) Element mapping (b) and EDX analyses (c) of the microcrystal shown in (a). The atomic ratio of $\mathrm{Cs}: \mathrm{Pb}: \mathrm{Br}$ is $\sim 2.9: 1: 5.7$. 

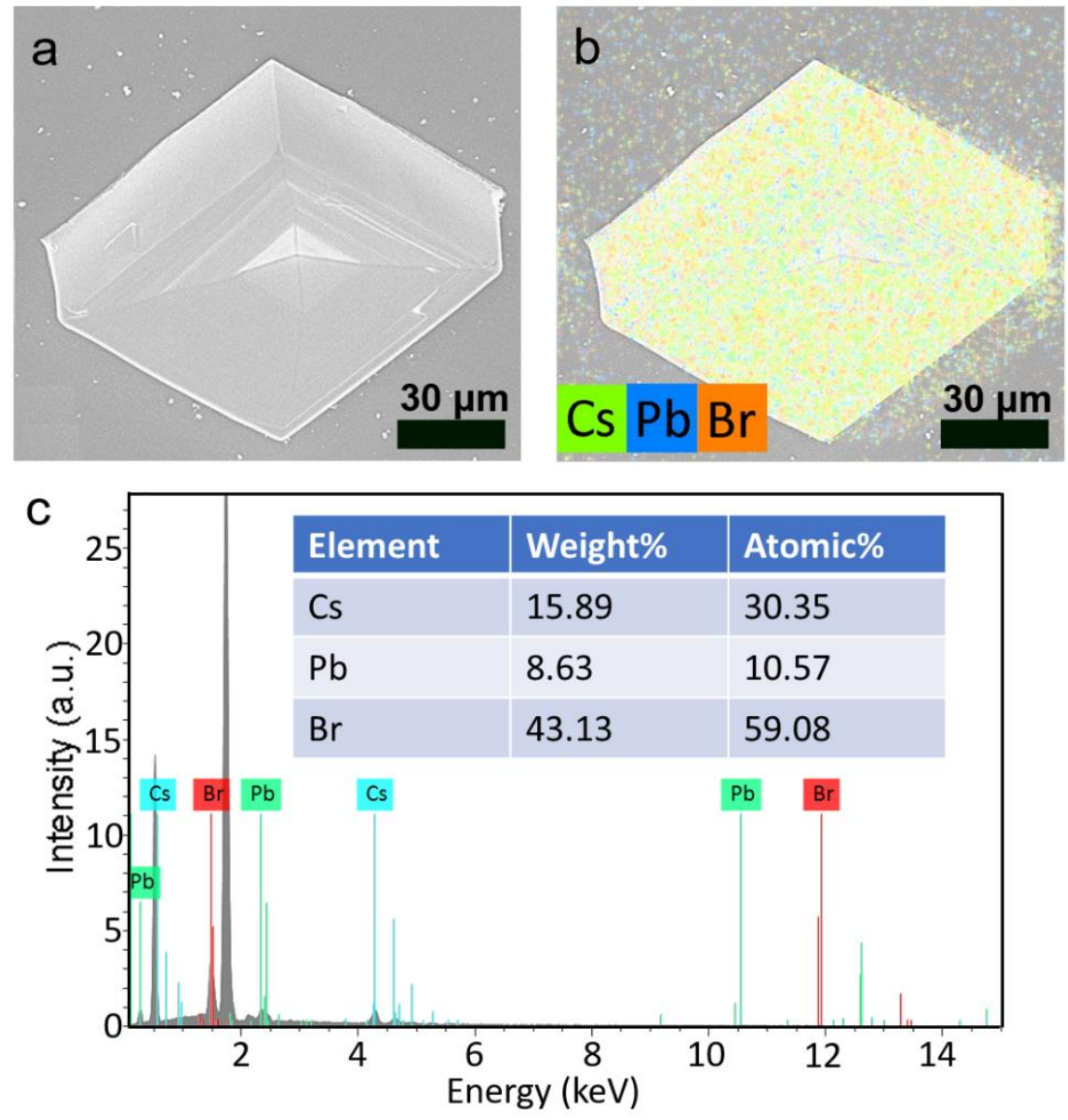

Figure S2. (a) SEM image of a selected $\mathrm{Cs}_{4} \mathrm{PbBr}_{6}$ microcrystal. (b, c) Element mapping (b) and EDX analyses (c) of the microcrystal shown in (a). The atomic ratio of Cs: $\mathrm{Pb}: \mathrm{Br}$ is $\sim 2.9: 1: 5.6$. 

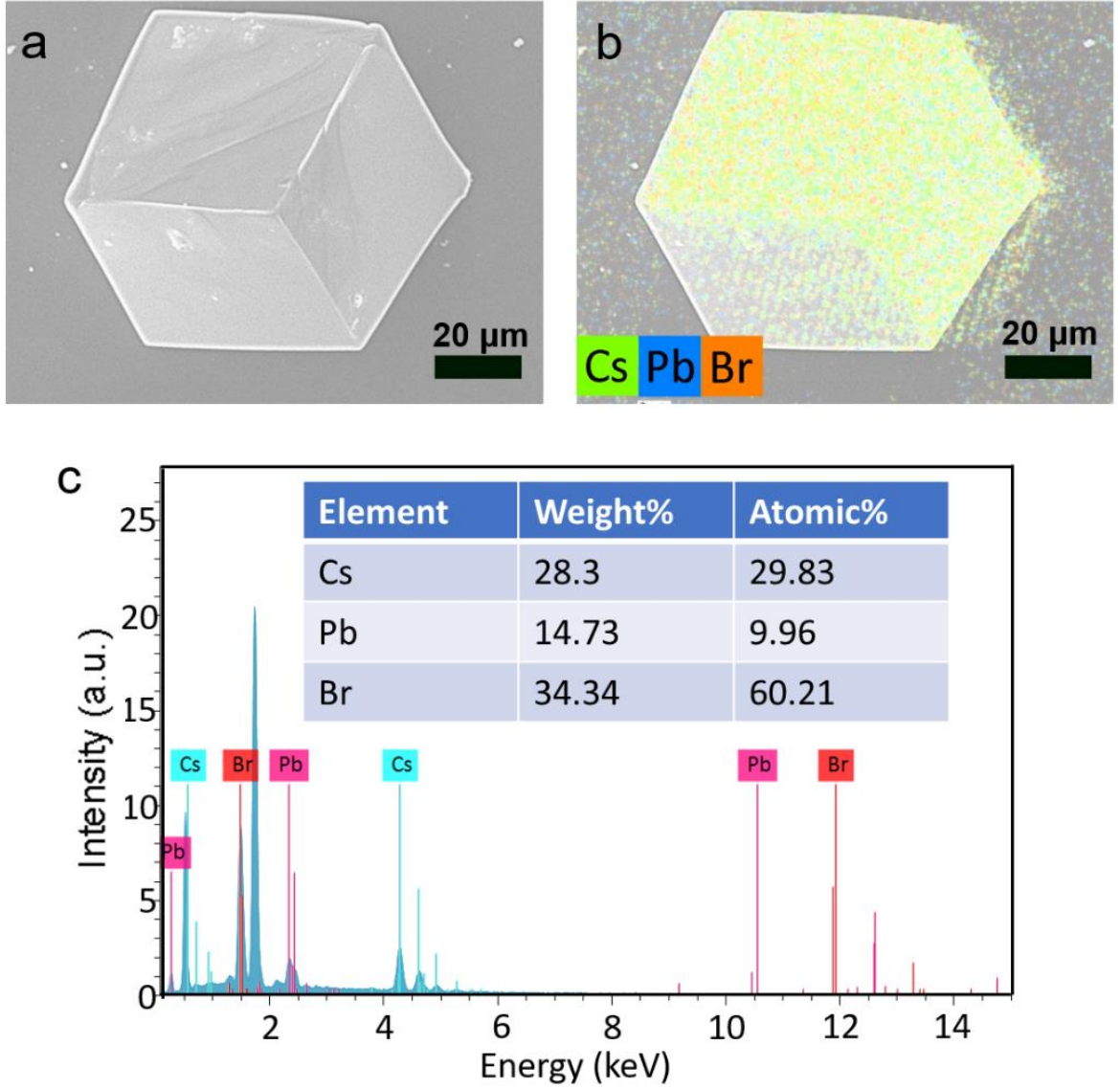

Figure S3. (a) SEM image of a selected $\mathrm{Cs}_{4} \mathrm{PbBr}_{6}$ microcrystal. (b, c) Element mapping (b) and EDX analyses (c) of the microcrystal shown in (a). The atomic ratio of $\mathrm{Cs}: \mathrm{Pb}: \mathrm{Br}$ is $\sim 3: 1: 6$. 

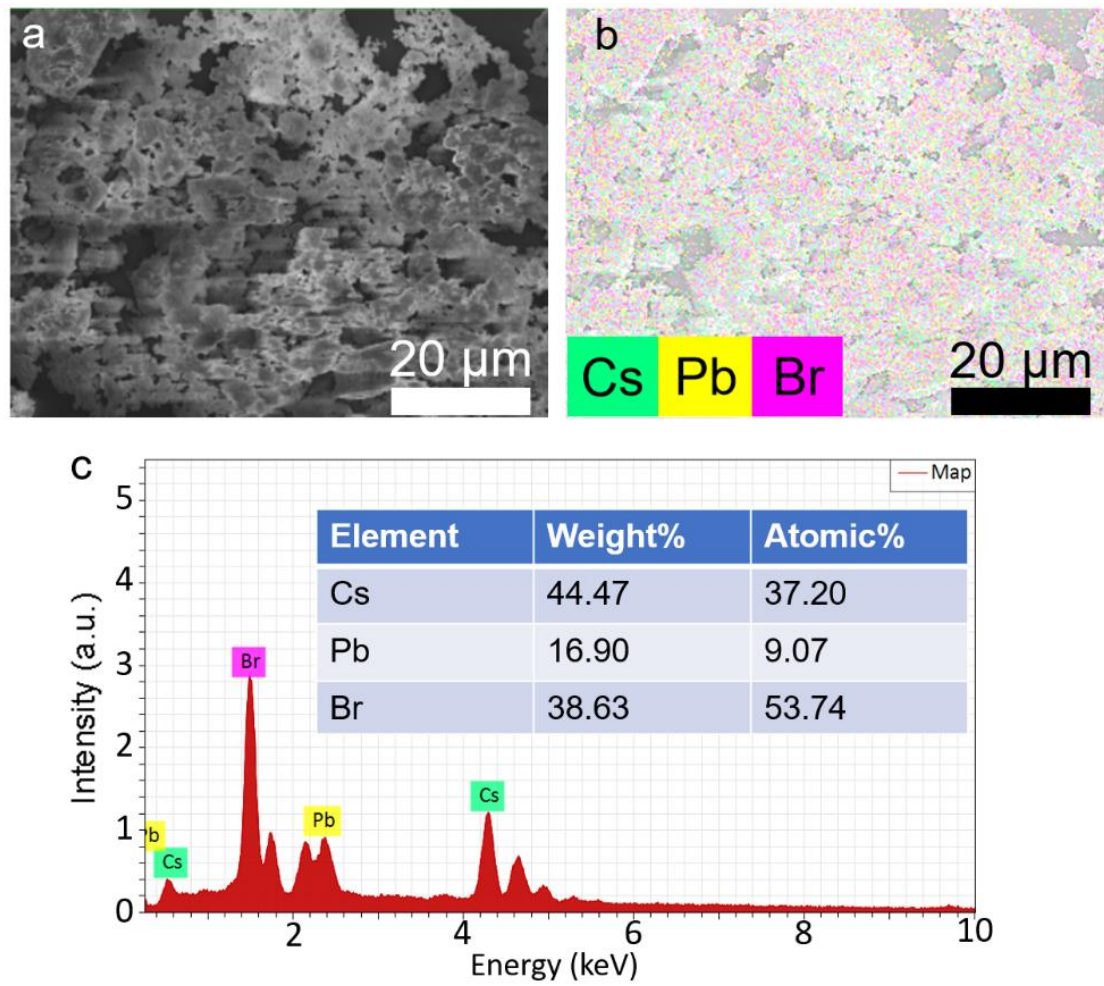

Figure S4. (a) SEM image of ground $\mathrm{Cs}_{4} \mathrm{PbBr}_{6}$ powders. (b, c) Element mapping (b) and EDX analyses (c) of the powders shown in (a). The atomic ratio of $\mathrm{Cs}: \mathrm{Pb}: \mathrm{Br}$ is $\sim 4.1: 1: 5.93$. 

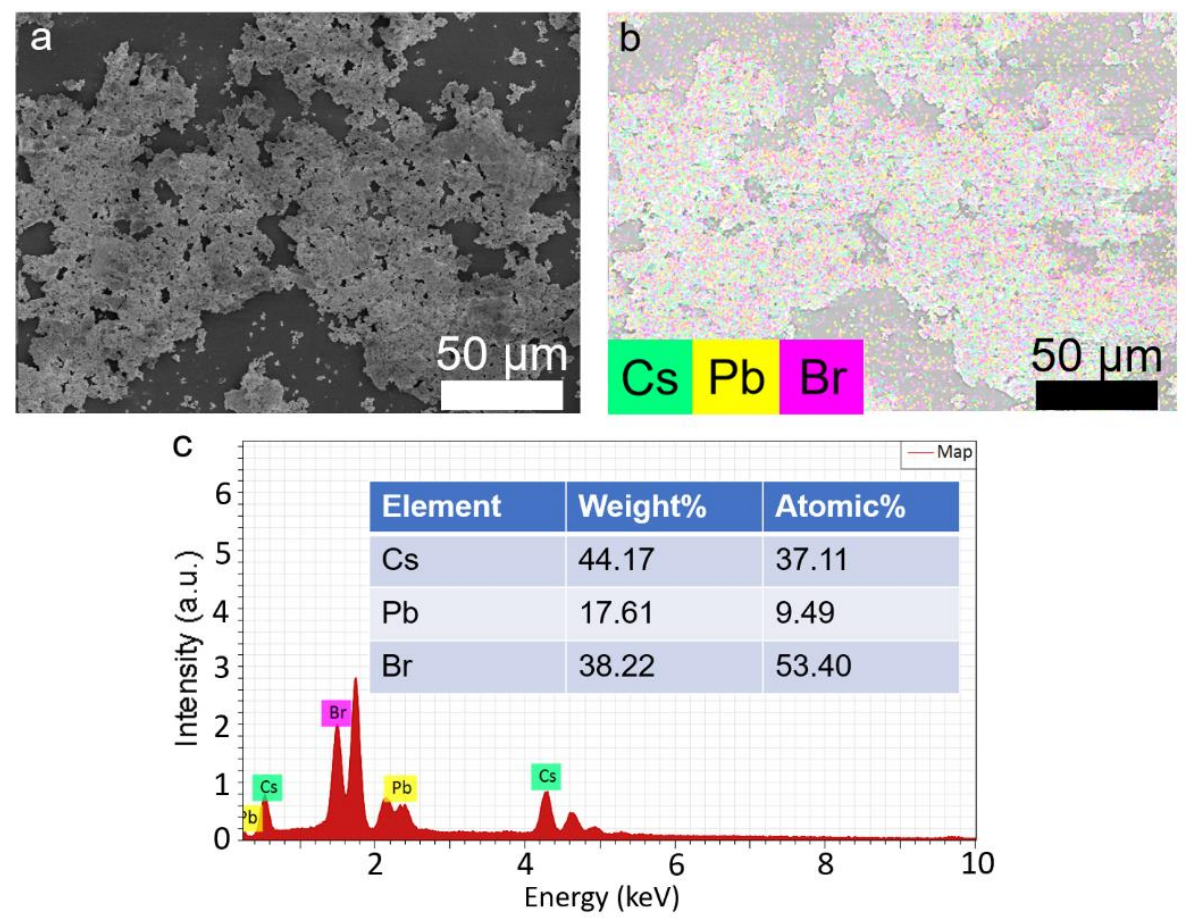

Figure S5. (a) SEM image of ground $\mathrm{Cs}_{4} \mathrm{PbBr}_{6}$ powders. (b, c) Element mapping (b) and EDX analyses (c) of the powders shown in (a). The atomic ratio of $\mathrm{Cs}: \mathrm{Pb}: \mathrm{Br}$ is $\sim 3.91: 1: 5.63$. 

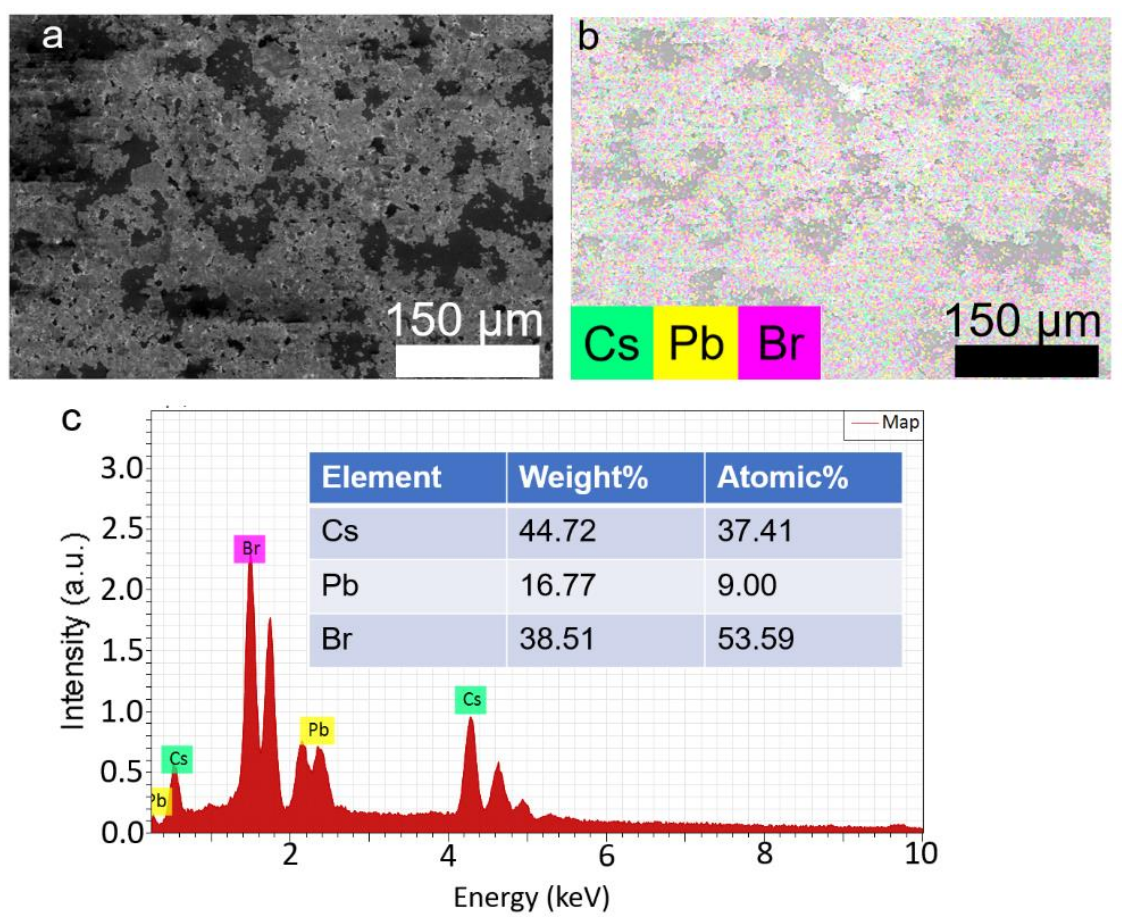

Figure S6. (a) SEM image of ground $\mathrm{Cs}_{4} \mathrm{PbBr}_{6}$ powders. (b, c) Element mapping (b) and EDX analyses (c) of the powders shown in (a). The atomic ratio of $\mathrm{Cs}: \mathrm{Pb}: \mathrm{Br}$ is $\sim 4.16: 1: 5.95$. 


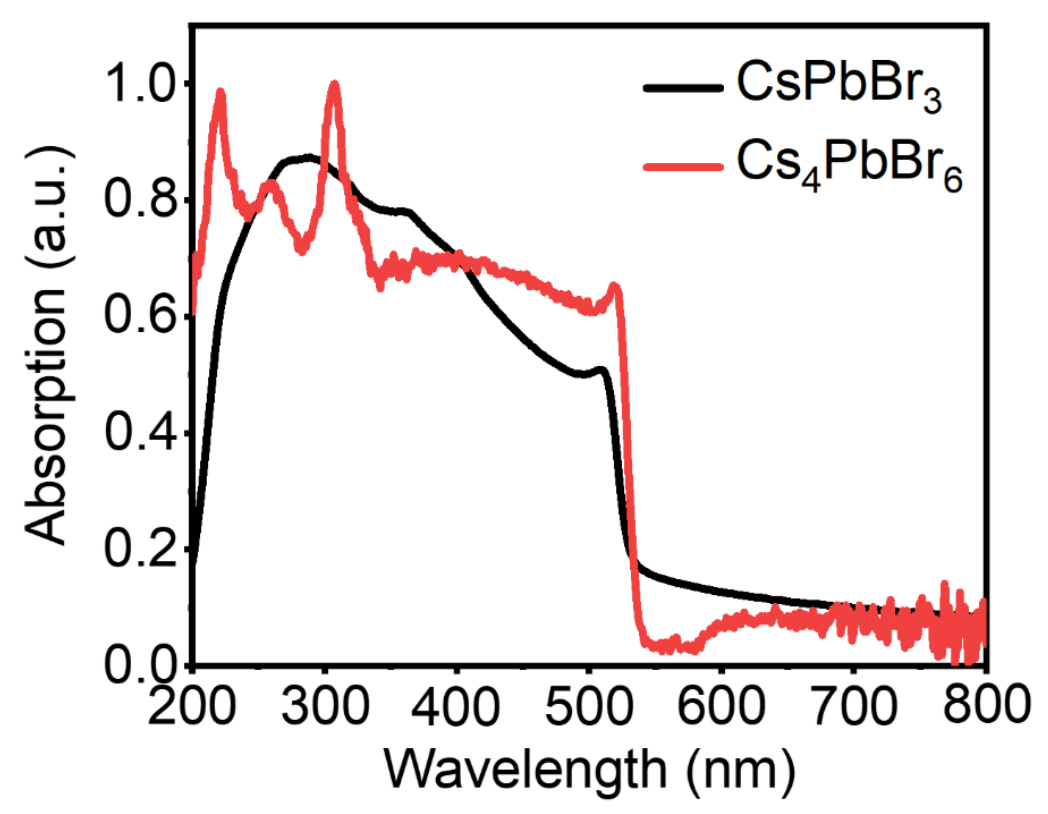

Figure S7. Comparison of optical absorption spectra between $\mathrm{CsPbBr}_{3}$ (black) and $\mathrm{Cs}_{4} \mathrm{PbBr}_{6}$ (red). Both samples were fabricated by drop-casting colloidal nanocrystals on the glass substrate. The $\mathrm{CsPbBr} 3$ colloids are a commercial product with an average size of $10 \mathrm{~nm}$ (Nanjing MKNANO Tech. Co., Ltd., Nanjing). Apparently, the two samples exhibit distinct optical absorption feature. That is, the optical absorption spectrum of the $\mathrm{CsPbBr}_{3}$ sample does not exhibit narrow peaks as observed in $\mathrm{Cs}_{4} \mathrm{PbBr}_{6}$. 


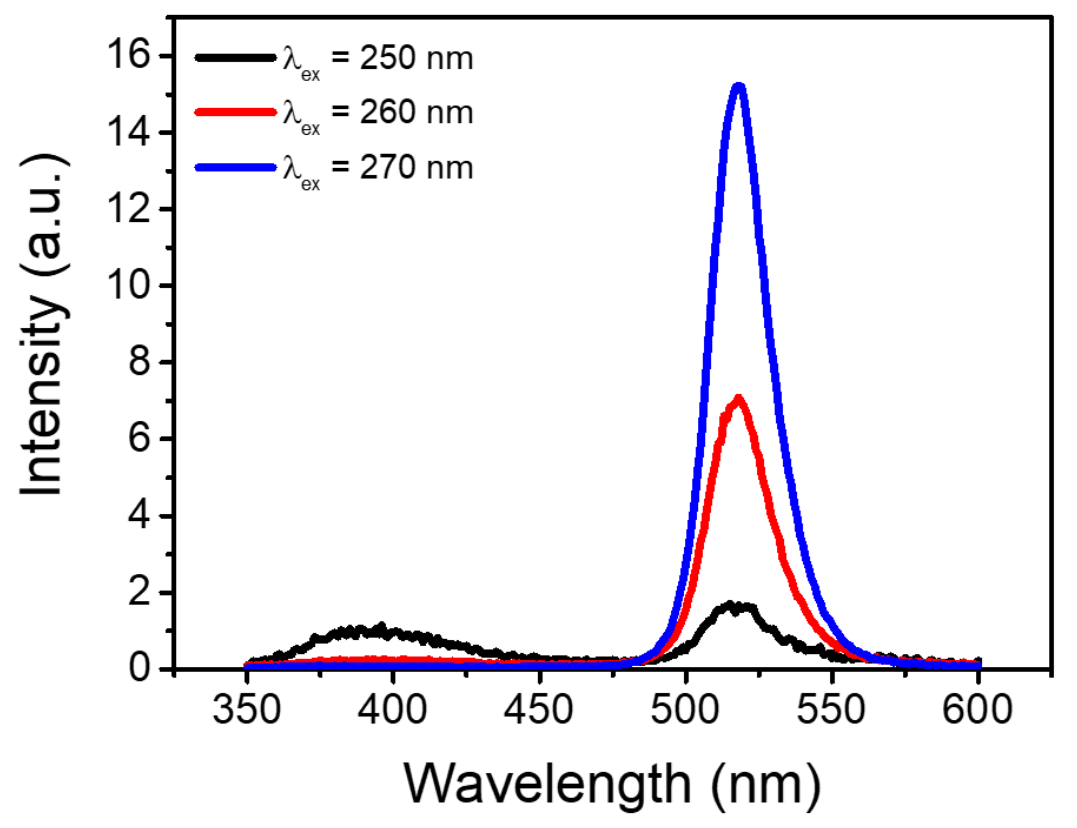

Figure S8. PL spectra recorded when the excitation wavelength is 250 (black curve), 260 (red curve), and $270 \mathrm{~nm}$ (blue curve). The UV PL band centered at $\sim 381 \mathrm{~nm}$ can be clearly observed when the excitation wavelength is $250 \mathrm{~nm}$, which gradually diminishes when the excitation shifts to the longer wavelength. 

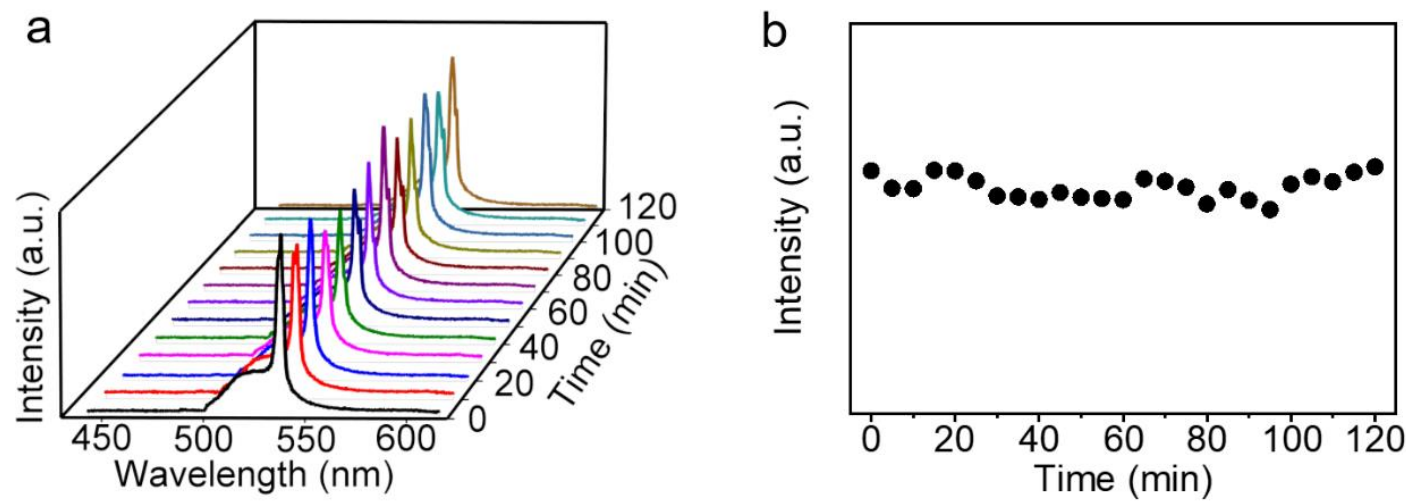

Figure S9. (a) Evolution of the PL spectra with the irradiation time by the picosecond laser $\left(490 \mathrm{~nm}, 25 \mathrm{ps}, 10 \mathrm{~Hz}\right.$ ). The pump fluence is $1.321 \mathrm{~mJ} / \mathrm{cm}^{2}$. (b) Plot of the PL intensity on the irradiation time. The results suggest the high photostability of $\mathrm{Cs}_{4} \mathrm{PbBr}_{6}$ microcrystals. 

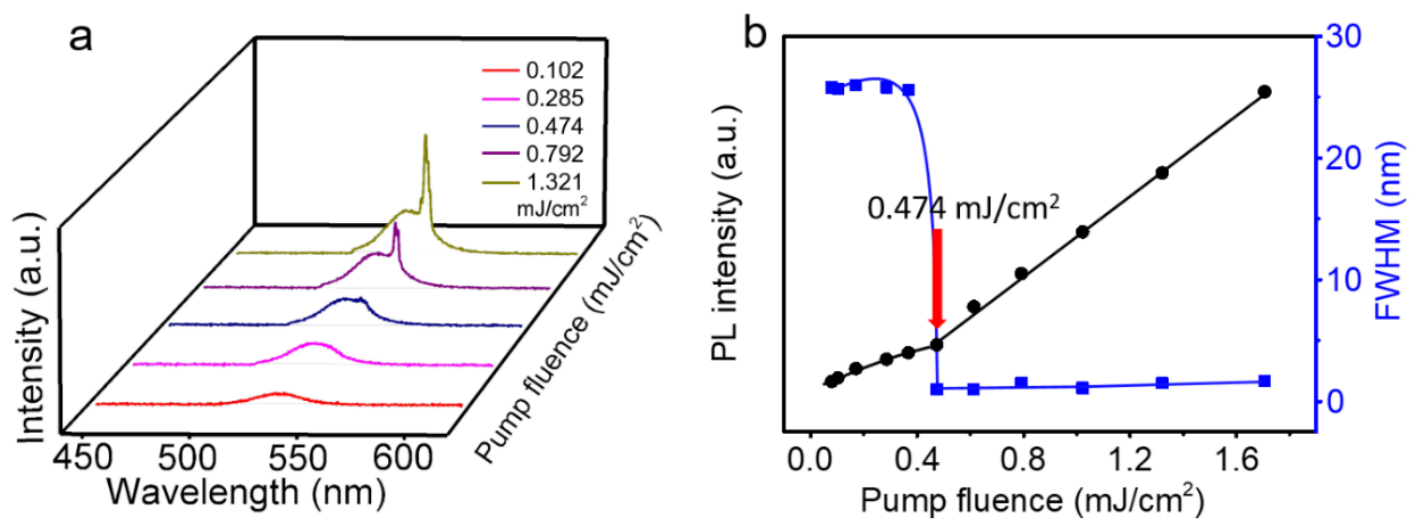

Figure S10. (a) Evolution the PL spectra with the pump fluence ranging from 0.102 to $1.321 \mathrm{~mJ} / \mathrm{cm}^{2}$ for the $\mathrm{Cs}_{4} \mathrm{PbBr}_{6}$ microcrystal with the edge length of $\sim 50 \mu \mathrm{m}$. (b) Plots of the PL intensity (black circles) and FWHM (blue squares) on the pump fluence. The discrete points are experimental data, while the solid lines are for convenience of eye vision. The red arrow shows the critical pump fluence above which lasing starts to occur. 

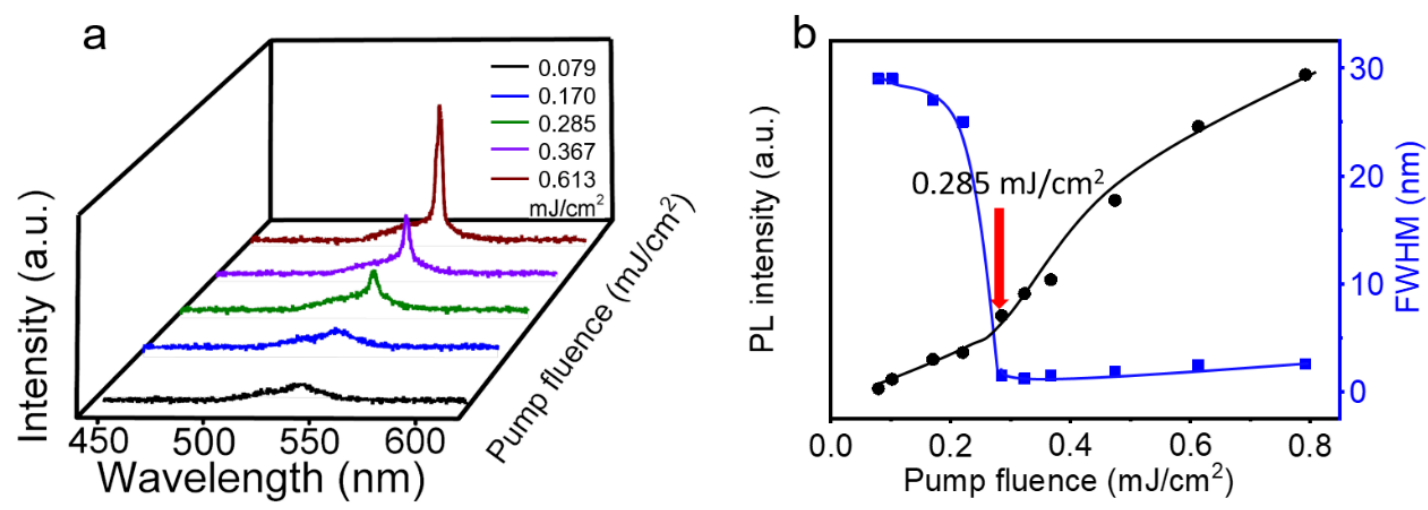

Figure S11. (a) Evolution the PL spectra with the pump fluence ranging from 0.079 to $0.613 \mathrm{~mJ} / \mathrm{cm}^{2}$ for the $\mathrm{Cs}_{4} \mathrm{PbBr}_{6}$ microcrystal with the edge length of $\sim 25 \mu \mathrm{m}$. (b) Plots of the PL intensity (black circles) and FWHM (blue squares) on the pump fluence. The discrete points are experimental data, while the solid lines are for convenience of eye vision. The red arrow shows the critical pump fluence above which lasing starts to occur. 

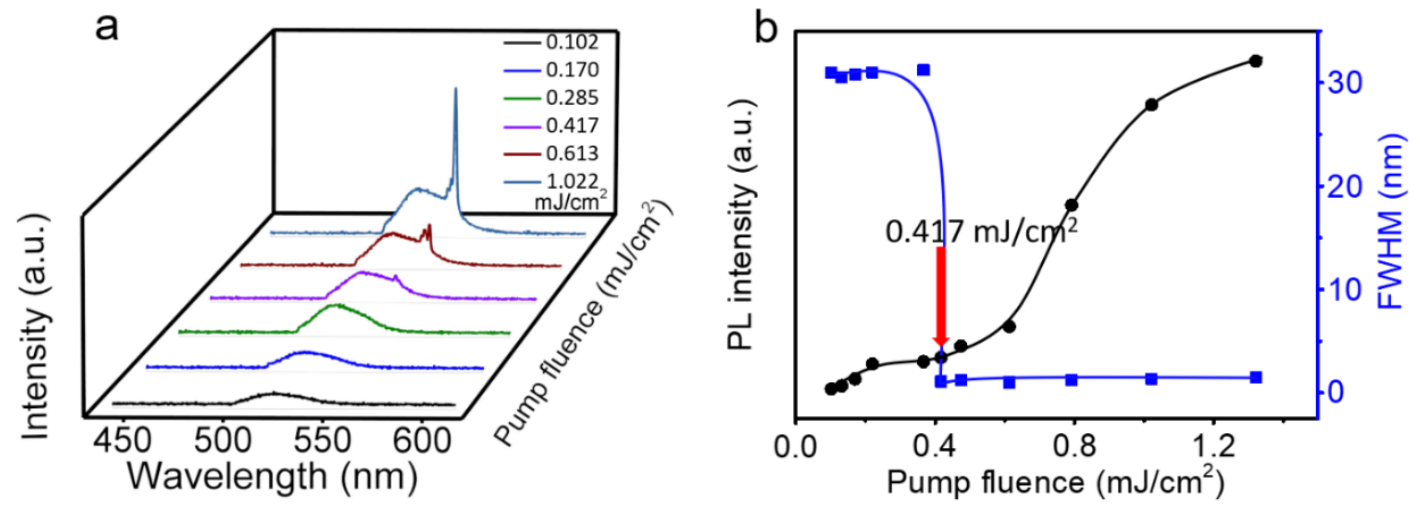

Figure S12. (a) Evolution the PL spectra with the pump fluence ranging from 0.102 to $1.022 \mathrm{~mJ} / \mathrm{cm}^{2}$ for the $\mathrm{Cs}_{4} \mathrm{PbBr}_{6}$ microcrystal with the edge length of $\sim 15 \mu \mathrm{m}$. (b) Plots of the PL intensity (black circles) and FWHM (blue squares) on the pump fluence. The discrete points are experimental data, while the solid lines are for convenience of eye vision. The red arrow shows the critical pump fluence above which lasing starts to occur. 